\title{
Performance Evaluation Of Machine Learning Techniques On Rpas Remote Sensing Images
}

\author{
R. Madanamohana, P Nagarjunapitty, K.Rishitha
}

\begin{abstract}
Recent advancements in remote sensing platforms from satellites to close-range Remotely Piloted Aircraft System (RPAS), is principal to a growing demand for innovative image processing and classification tools. Where, Machine learning approaches are very prevailing group of data driven implication tools that provide a broader scope when applied to remote sensed data. In this paper, applying different machine learning approaches on the remote sensing images with open source packages in $R$, to find out which algorithm is more efficient for obtaining better accuracy. We carried out a rigorous comparison of four machine learning algorithms-Support vector machine, Random forest, regression tree, Classification and Naive Bayes. These algorithms are evaluated by Classification accurateness, Kappa index and curve area as accuracy metrics. Ten runs are done to obtain the variance in the results on the training set. Using $k$-fold cross validation the validation is carried out. This theme identifies Random forest approach as the best method based on the accuracy measure under different conditions. Random forest is used to train efficient and highly stable with respect to variations in classification representation parameter values and significantly more accurate than other machine learning approaches trailed.
\end{abstract}

Keywords: - Remote Sensing, Machine Learning, $R$ software, Support Vector Machine, Random Forest, Classification and Regression Tree, Naive Bayes

\section{INTRODUCTION}

Because of the gigantic expansion in proportion of remote distinctive stages, beginning from satellites to short region Remotely Piloted Aircraft System (RPAS), is head to a making energy for inventive picture preparing and plan instruments. This work studies a similarity of the Support Vector Machine (SVM), Random Forest (RF), Naive Bayes and Classification and Regression Tree (CART) AI tallies utilizing open source $\mathrm{R}$ packs for expelling locale use classes in RPAS chose orthomosaic. A modified inductive way to deal with oversee see structures in the information by utilizing AI estimations. To make wants for information driven assembling and fall away from the faith planning

plan affiliations are related with close information. $\mathrm{R}$ contains predefined packages using which we implemented this algorithms on the data. The dataset used in this approach contains the data about Gulfport images [2]. The

Revised Manuscript Received on August 14, 2019.

R. Madanamohana, Professor, Department of Computer Science and Engineering, Bharat Institute of Engineering and Technology, Hyderabad, Telangana, India(email id: madanmohanr@biet.ac.in)

P Nagarjunapitty, Senior Scientific Officer, Indian Institute of Science, Bangalore, India(email id: nagarjuna@iisc.ac.in)

K. Rishitha, UG Scholar, Department of Computer Science and Engineering ,Bharat Institute of Engineering and Technology, Hyderabad, Telangana, India(email id: kogantirishitha@gmail.com)
$\mathrm{R} / \mathrm{r}$ miner package [3] is an aggregator of fifteen regression and fourteen classification methods. It furthermore includes approaches designed to determine accuracy metrics over results [1]. The classification performance of these algorithms is tested on training sets of various data size. Metrics such as classification accuracy, kappa index and curve area have been applied to estimate the performance of SVM, RF, CART and Naive Bayes algorithms. Validation for these algorithms using k-fold cross validation. Ten folds have been applied on the data of training and testing to evaluate the variance in the results.

\section{RELATED WORK}

Papageorgiou EI et al., have applied fuzzy cognitive map based approach as a basis for decision support system in precision agriculture application for predicting defer in cotton crop production in 2011 [4]. Their work evaluates the method of yield want in cotton crop creation subject to the woolen academic maps of delicate figuring technique. Padded Cognitive Map (FCM) is a mix of warm reason and mental guide speculations, and is utilized for appearing and importance experts' learning. It is fit for supervising conditions including sketchy depictions utilizing relative system, for example, human thinking does. The researched method assessed for 360 cases surveyed during the time of six coming about years (2001-2006) in a fundamental cotton field, in envisioning the yield class between two potential portrayals ("low" and "high"). The picked up results reveal its relative favoured position over the benchmarking AI calculations worked for equivalent edifying aggregation for the length referenced by given that choices that matches better with the genuine assessed ones. The basic improvement of this methodology is its key structure and flexibility, tending to information evidently and considerably more clearly. Along these lines, it may be a gainful mechanical get together in imagining cotton yield and improving harvest the overseers. Zheng YJ et al. given Multi target sparklers improvement in oil harvest age for variable rate course of action in 2013 [5]. Additionally, in addition for oil crop preparation demonstrated a multi target redesign issue model, which contemplates procure yield and quality similarly as centrality use and trademark impacts. Propose a cream Multi Objective Fireworks Optimization Algorithm (MOFOA) for proficiently managing the issue that develops a lot of answers for the Pareto ideal front by mirroring the effect of sparklers. By utilizing the likelihood of Pareto control for individual assessment and choice, and

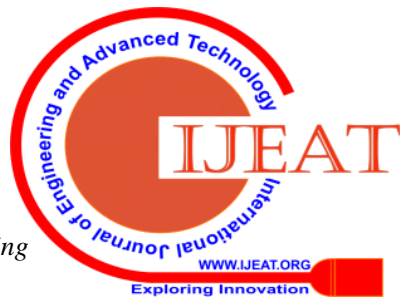




\section{PERFORMANCE EVALUATION OF MACHINE LEARNING TECHNIQUES ON RPAS REMOTE SENSING IMAGES}

joins differential progression (DE) officials to develop data sharing among the people. The test tests and true applications in oil crop creation in East China exhibit the sufficiency and reasonableness of the calculation.

Hung $\mathrm{C}$ et al. utilized a methodology subject to Feature learning for weed assembling utilizing high targets ethereal pictures from a pushed camera mounted on a UAV Remote Sensing in 2014 [6]. The improvement of immaterial effort Unmanned Aerial Vehicles (UAVs) and light weight imaging sensors has acknowledged fundamental vitality for their utilization for remote distinctive applications. So also proposed an elective learning-based method utilizing highlight comprehending how to restrict the manual exertion required. Reviewed thusly to oversee weed assembling on three weeds of hugeness in Australia: water hyacinth, serrated tussock and tropical soft drink apple. This outcomes demonstrated that social affair pictures at 5 to $10 \mathrm{~m}$ understood the most huge classifier precision, surveyed by F1 scores of up to $94 \%$.

\section{DATA}

The MUUFL Gulfport dataset [2] collection contains LiDAR and co registered hyper spectral data over the campus. The ground truth map is provided by manually labelling the pixels into the scene by the following classes in the scene: mostly-grass ground surface, trees, dirt and sand, mixed ground surface, road, water, buildings, shadow of buildings, yellow curb, sidewalk, cloth panels (targets), and unlabeled points. Google Maps, Google Earth and geotagged photographs at the scene were used to assist the labelling process. The base MUUFL Gulfport data set-1 scene contains $325 \times 337$ pixels over 72 bands resulting in a new hyper spectral image of 64 bands. The lower right corner of the original image contains invalid area, thus only the first 220 columns were used for the ground truth mapping. The size of the cropped hyper spectral imagery is $325 \times 220 \times 64$. RGB image of the new data set (campus 1 scene) used for ground truth mapping is showing in Figure 1 .

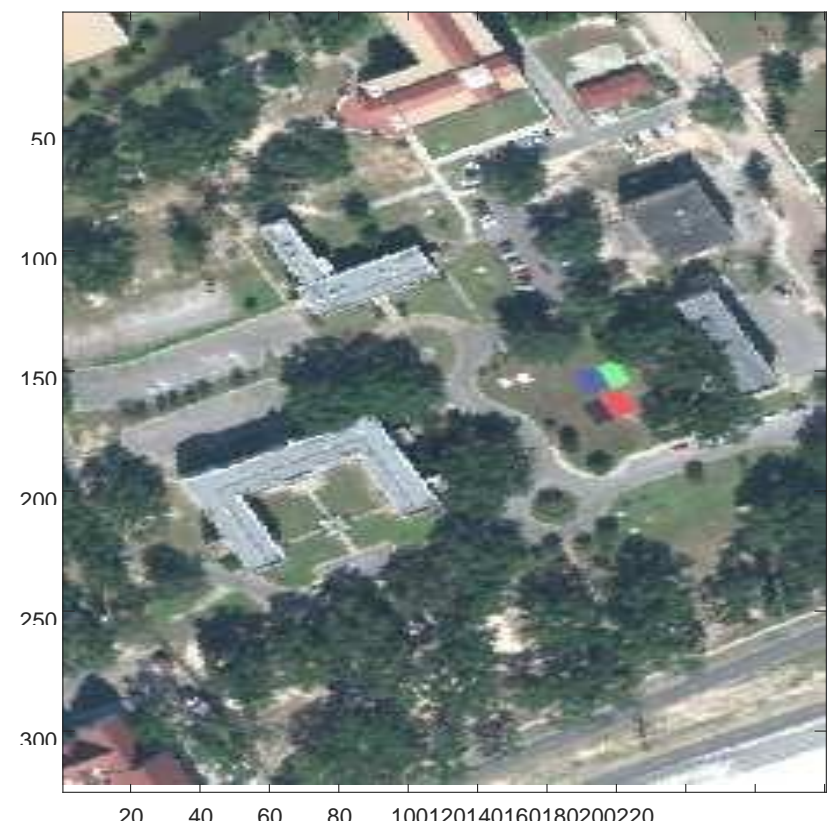

Figure1: Cropped MUUFL Gulfport campus 1 hyperspectral imagery RGB image.

Figure 2 shows the high-level labels for each of the following twelve class in the scene: trees (label"1"), mostlygrass ground surface (label " 2 "), mixed ground surface (label "3"), dirt and sand (label "4"), road (label "5"), water (label "6"), buildings (label "7"), shadow of buildings (label "8”), sidewalk (label "9"), yellow curb (label "10"), cloth panels (targets) (label "11"), and unlabeled points (label "1 "). Specifically, "mostly-grass ground surface" class refers to ground surface that are visually identifiable as covered in grass, while "mixed ground surface" class refers to groundcovering materials that may contain grass but may also contain soil, dirt and any other surface material. Cloth panels with various spectral characteristics were placed in the scene to construct super-pixel and sub-pixel targets. Only those that are larger than pixel-level and visually identifiable are labelled separately into "cloth panels" class. All other class may contain sub-pixel cloth targets. This new cropped data set consists of fifty cloth panels. Specific target ground truth locations can be found in the label MATLAB structure file. All points that are un-identifiable as any of the first eleven classes are labelled as "unlabeled points".

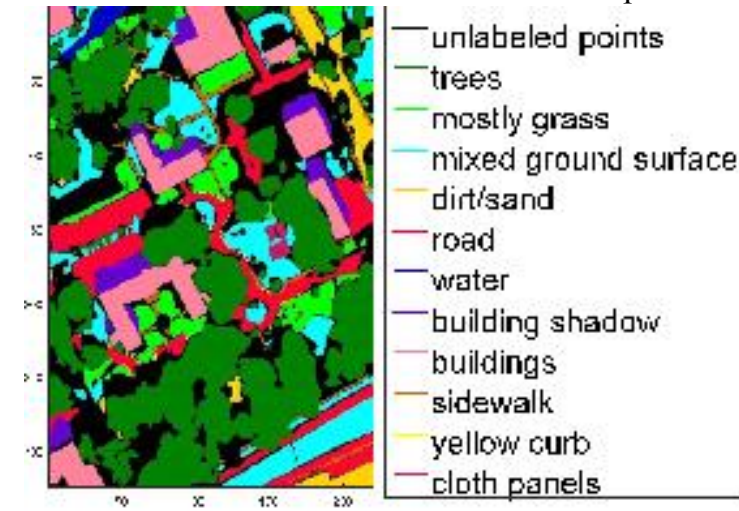

Figure2: MUUFL Gulfport campus 1 hyperspectral imagery scene labels ground truth map.

\section{METHODOLOGY}

\section{A. Pre-processing}

Firstly, the data which we have taken should be preprocessed to find out if there are any missing values present in it. R provides any $\mathrm{NA}()$ function to check the missing values in the data. If there are missing values then we can remove them using omit() function.

\section{B. Classification model training}

By performing data slicing the dataset is divided into training and testing sets and $\mathrm{K}$-fold cross validation technique is applied on the data and accuracy metrics are founded for machine learning algorithms. Based on maximum mean accuracies ensuing from ten-fold (10-fold) cross-validation optimal parameters are selected. Trained MLA classification models utilizing picked parameters all things considered blueprint of tests before gauge examination. The gatherings utilized for applying the four 
machine learning algorithms are caret, e1071, rpart and random forest.

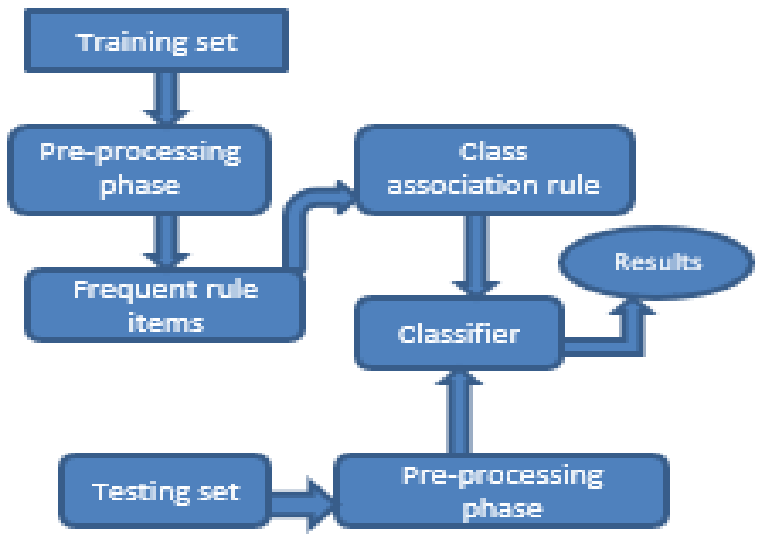

Figure3: Proposed Model

\section{Support Vector Machine (SVM)}

A SVM is a classifier that pieces its information space into two districts, isolated by a straight limit. Here, it has grasps how to see high complexity circles. SVM is Robust or more generalize.

Essential SVM estimation is a competent parallel classifier. The thought behind SVM way to deal with oversee SLR is that we map our information to a segment space. This segment space is the reason behind the SVM figuring which picks a straight choice surface (hyper plane) utilizing the game-plan of checked information inside it. This surface is then used to depict future occasions of information. Information is amassed dependent on which side of the choice surface it falls. SVM is critical to both straight particular and non-authentically discernible models. Models not straight obvious are changed utilizing piece works a mapping limit, into direct distinguishable ones. It will all in all be described as looks for after. The ideal hyper plane keeping the two classes can be tended to as given in condition (1):

$$
w \cdot X+\beta=0
$$

Where, X-sample input vector defined as

$$
\begin{gathered}
\left\{\left(x_{1}, y_{1}\right),\left(x_{2}, y_{2}\right), \ldots \ldots . .\left(x_{k}, y_{k}\right)\right\}, \\
x_{k} \in R_{n}, y_{k} \in\{1,-1\}
\end{gathered}
$$

Where the propensity part.

The dealt with pair $\langle x, y\rangle$ is the depiction of every datum used to shape hyper plane which are $\mathrm{N}$-dimensional vectors set apart with separating y are given in conditions (2) and (3).

$$
\begin{gathered}
w . X+\beta \geq 1 \quad \text { if } \text { yi }=1 \\
w . X+\beta \leq-1 \quad \text { if } \mathrm{yi}=-1
\end{gathered}
$$

These could be combined into one set of inequalities is given in equation (4):

$$
y_{i}\left(x_{i} \cdot w+\beta\right) \geq 1
$$

The above unbalanced properties hold for all information tests (direct particular and work the perfect hyper plane condition). The perfect hyper plane is the groundbreaking one which confines the game-plan data with a maximal edge. One of the including detachment between the parallel and multi class SVM is the set $\mathrm{y}=\{1,2,3, \mathrm{k}\}$ and rehearses which are committed to this set. Packages used: e1071, caret.

e1071: used for short time Fourier transforms latent class analysis, support vector machines, naive Bayes classifier, fuzzy clustering, bagged clustering and shortest path computation.

Caret: Miscellaneous breaking points concerning getting ready and plotting methodology fall away from the confidence models. The caret pack (short for classification and drop into wrongdoing orchestrating) contains abilities to streamline the model getting ready system for complex lose the confidence and classification issues. The get-together "proposes" field blends 27 packs. Caret burdens packages as required and perceive that they are shown.

\section{Random Forests}

Sporadic Forests (RF), made by Bremen, is a get-together solicitation plot that uses a bigger part vote to envision classes dependent on the heap of information from different choice trees. RF makes various trees by abstractly sub setting a predefined number of factors to part at each focal point of the choice trees and by stowing. Sacking produces preparing information for each tree by testing with substitution various models equivalent to the measure of tests in the source dataset. RF finishes the Gini Index to pick a "best split" limit of information respects for given classes. The Gini Index restores a degree of class parent focus.

\section{Packages used: random Forest}

Random Forest- implements Bremen's random forest algorithm (based on Breiman and Cutler's original Fortran code) for classification and regression. It can also be used in unsupervised mode for assessing proximities among data points.

\section{E. Naive Bayes}

Artless Bayes (NB) [8] is an outstanding accurate learning computation recommended as a base level classifier for connection with various counts. NB assessments class unanticipated probabilities by "sincerely" expecting that for a given class the information sources are free of each other. This doubt yields a partition most distant point shown by the results of the joint probabilities that the classes are certifiable given the data sources. NB reduces the issue of isolating classes to finding class prohibitive minor densities, which address the probability that a given model is one of the possible target classes. NB performs well against various choices aside from if the data contains related information sources. 


\section{PERFORMANCE EVALUATION OF MACHINE LEARNING TECHNIQUES ON RPAS REMOTE SENSING IMAGES}

\section{F. Classification and Regression tree}

Decision Trees are dependably used in data mining with the objective of making a model that predicts the estimation of a goal (or ward variable) in light of the estimations of a couple of information (or self-controlling segments). Gettogether and Regression Trees [9] or CART for short is a term agreeable by Leo Breiman with propose Decision Tree figuring's that can be used for sales or fall away from the confidence farsighted exhibiting issues. As a rule, this estimation is suggested as "decision trees", yet on unequivocal stages like $\mathrm{R}$ they are proposed by the more present day term CART. In the Depiction Trees, the objective variable is unmitigated and the tree is utilized to see the "class" inside which an objective variable would no doubt fall into. Where in the Regression tree the objective variable is driving forward and tree is utilized to anticipate its worth.

\section{Packages used: rpart}

Rpart- The rpart adventures produce classification or fall away from the faith models of a general structure utilizing a two phase approach; the subsequent models can be tended to as parallel trees.

\section{EXPERIMENTAL RESULTS}

The accuracy metrics are reported in three boxplots (Fig: $3,4,5)$ which represents respectively the classification accuracy (Acc), kappa index $(\mathrm{K})$ and area under curve (AUC) for K-fold cross validation that is being carried out.

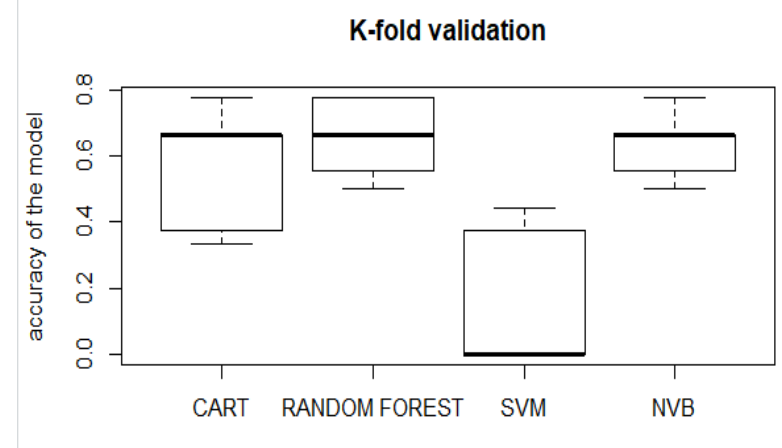

algorithm

Figure4: Boxplot of classification accuracy calculated for K-fold cross validation

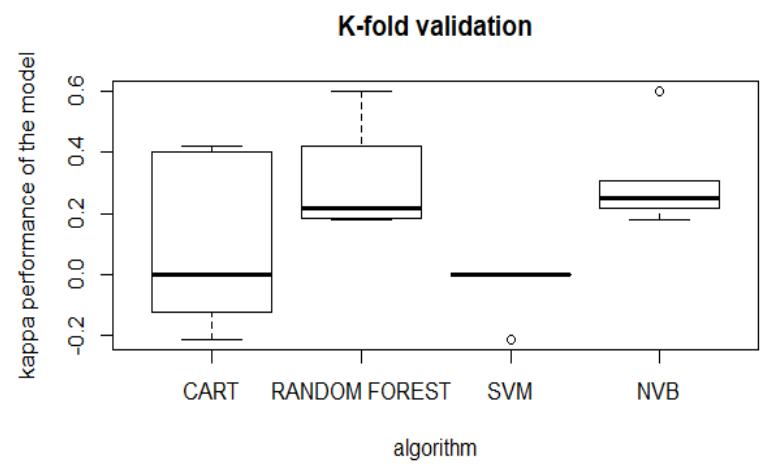

Figure5: Boxplot of kappa index calculated for K-fold cross validation

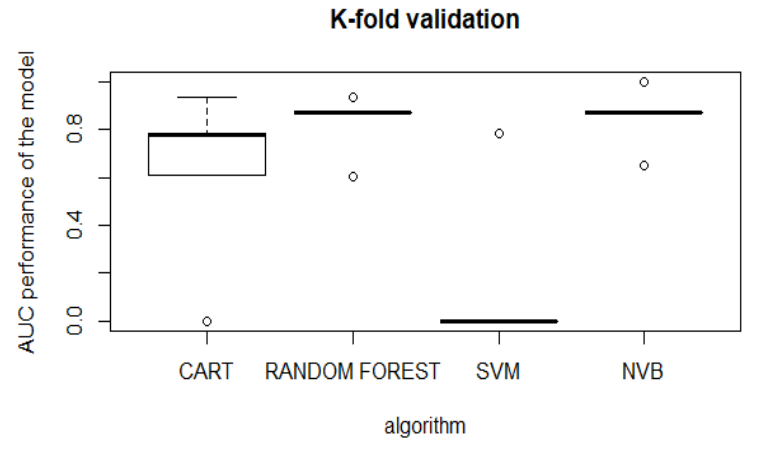

Figure6: Boxplot of area under curve calculated for K-fold cross validation

Halftestset validation

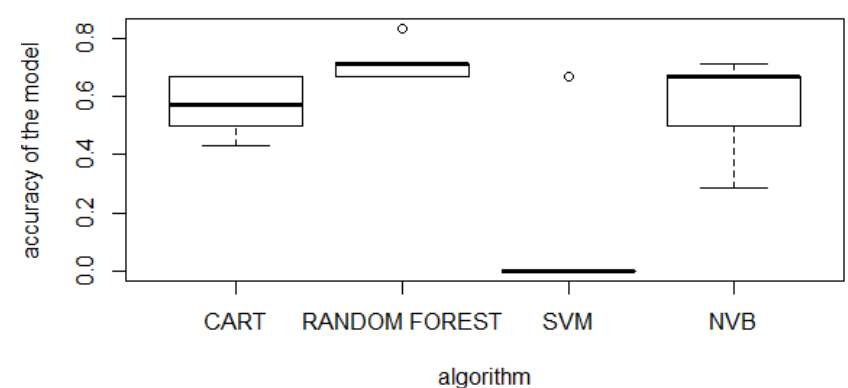

Figure7: Boxplot of classification accuracy calculated for half test set

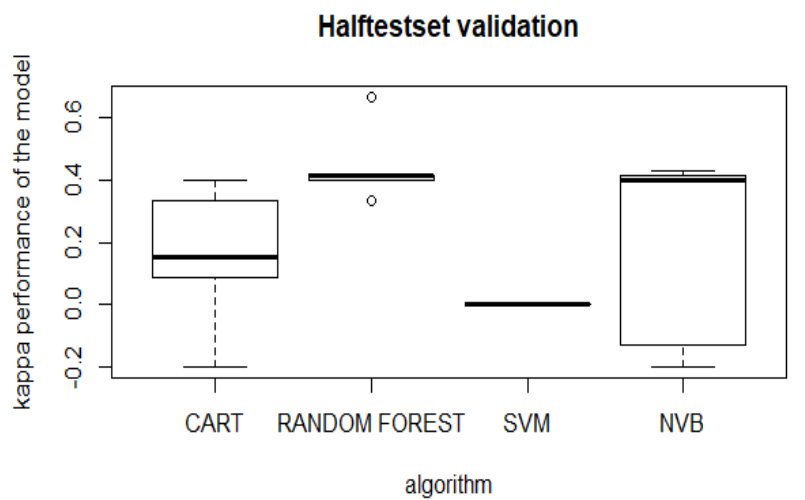

Figure8: Boxplot of kappa index calculated for half test set 


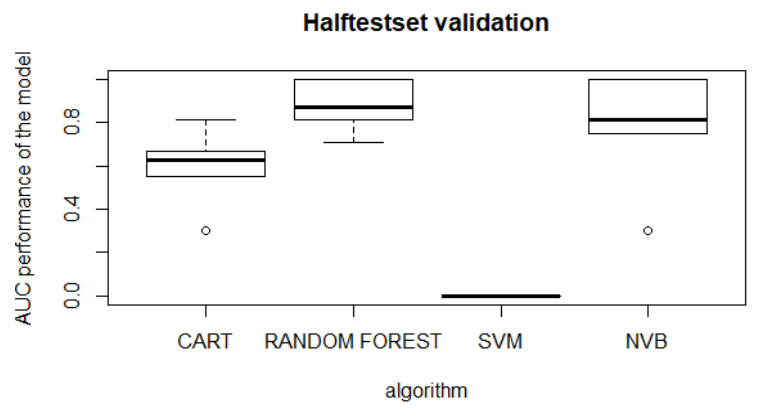

Figure9: Boxplot of area under curve calculated for half test set

Fulltestset validation

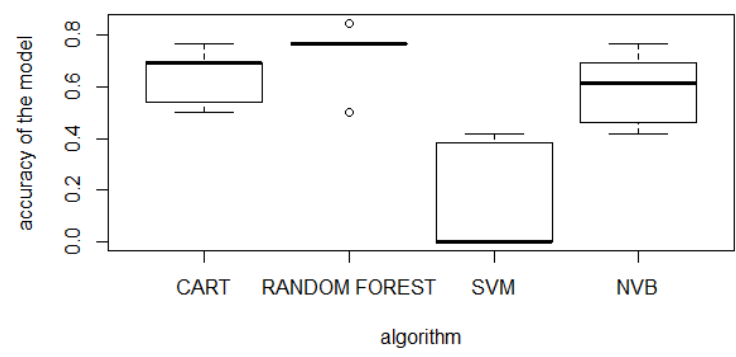

Figure10: Boxplot of arrangement precision calculated for Full test set

full test set

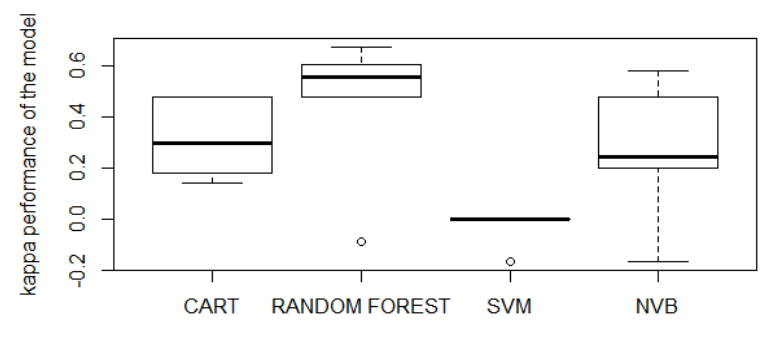

algorithm

Figure11: Boxplot of kappa index calculated for Full test set

full test set

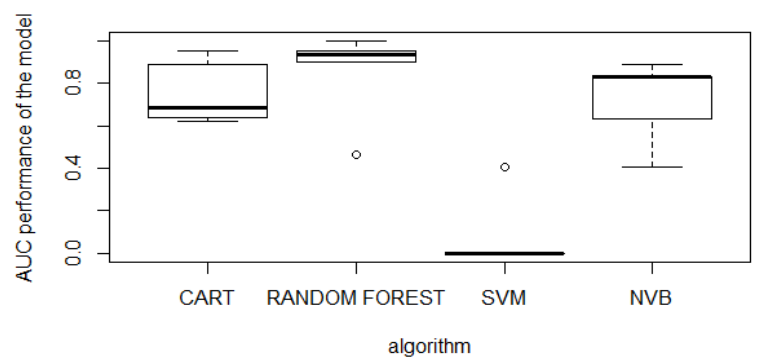

Figure12: Boxplot of area under curve calculated for Full test set

\section{CONCLUSION}

In this work, machine learning algorithms SVM, RF, CART and Naive Bayes of accuracy metrics such as have been obtained. Before justification was applied, according to accuracy metrics Random forest has given better results when compared to other. When $\mathrm{K}$-fold validation was applied the values varied for different sizes of training and testing sets. When training set is larger than testing set, better results were obtained for RF algorithm .Whereas, for half and full validation sets there was no variance in the results.

\section{FUTURE WORK}

The idea on future appraisals will test more AI counts joining unmistakable continues running with different blends of orchestrating and test sets to improve results. We can in like manner consider undeniable datasets, through cautious assurance of pixels for both planning and testing tests. Regardless of the way that by doing this the size of the dataset reduces, at any rate there is a legitimacy that it will give better information on the direct of the AI frameworks.

\section{REFERENCES}

1. Cortez P. (2010). Data Mining with Neural Networks and Support Vector Machines Using the R/rminer Tool. In: Perner P. (eds) Advances in Data Mining. Applications and Theoretical Aspects. ICDM 2010. Lecture Notes in Computer Science, vol 6171. Springer, Berlin, Heidelberg

2. Gader, P., Zare, A., Close, R., Aitken, J., \& Tuell, G. (2013). Muufl gulfport hyperspectral and lidar airborne data set. Univ. Florida, Gainesville, FL, USA, Tech. Rep. REP-2013-570.

3. Cortez, P. (2016). rminer: Data Mining Classification and Regression Methods. R package version, 1(2).

4. Papageorgiou, E. I., Markinos, A. T., \& Gemtos, T. A. (2011). Fuzzy cognitive map based approach for predicting yield in cotton crop production as a basis for decision support system in precision agriculture application. Applied Soft Computing, 11(4), 3643-3657.

5. Zheng, Y. J., Song, Q., \& Chen, S. Y. (2013). Multiobjective fireworks optimization for variable-rate fertilization in oil crop production. Applied Soft Computing, 13(11), 4253-4263.

6. Hung, C., Xu, Z., \& Sukkarieh, S. (2014). Feature learning based approach for weed classification using high resolution aerial images from a digital camera mounted on a UAV. Remote Sensing, 6(12), 12037 12054.

7. Brownlee, J. (2014). Machine learning mastery. URL: http://machinelearningmastery. com/discover-featureengineering-howtoengineer-features-and-how-togetgood-at-it.

8. Brownlee, J. (2014). Master Machine learning Algorithms.

9. Brownlee, J. (2014). Machine learning mastery.

\section{AUTHORS PROFILE}

R. Madanamohana: Professor, Department of Computer Science and Engineering, Bharat Institute of Engineering and Technology, Hyderabad, Telangana, India

Email id: madanmohanr@biet.ac.in

P Nagarjunapitty: Senior Scientific Officer, Indian Institute of Science, Bangalore

Email id: nagarjuna@iisc.ac.in

K.Rishitha:UG Scholar, Department of Computer Science and Engineering, Bharat Institute of Engineering and Technology, Hyderabad, Telangana, India

Email id: kogantirishitha@gmail.com 